\title{
RISQUE ET RENTABILITE
}

\author{
GÉrard Croset
}

France

1. M. Karl Borch a présenté, dans plusieurs de ses communications à l'ASTIN 1), un modèle de comportement de l'assureur, qu'il qualifie lui-même de simplifié, mais qui met néanmoins en évidence une intéressante propriété d'optimalité. L'assureur maximiserait la valeur actuelle des dividendes futurs en laissant les bénéfices éventuels s'ajouter aux fonds propres jusqu'au niveau $Z$; les montants qui porteraient alors les richesses libres au dessus du niveau $Z$ seraient intégralement distribués. L'espérance mathématique optimale $V(S, Z)$ de la valeur actuelle des dividendes futurs dépend, dans ces conditions, du capital $S$ initial. D'autre part, il reste entendu que si $S>Z$, alors $(S-Z)$ est immédiatement ,remboursé", et, si $S=0$, la société cesse, en principe, ses opérations, toutefois, M. Karl Borch a envisagé la possibilité d'un renflouement ${ }^{2}$ ).

2. L'utilisation pratique de ce modèle pose deux problèmes particulièrement importants:

- la société a-t-elle le droit de fonctionner avec le capital $S$ ?

- les capitalistes accepteraient-ils d'investir $S$ ?

2.1. Le premier de ces problèmes a été envisagé par nous-même dans une étude précédente ${ }^{3}$ ). Il apparaît que la contrainte imposée par l'autorité de tutelle peut-être sommairement résumée par l'obligation d'un capital minimum $T$. La valeur actuelle optimale $V\left(S, T, Z_{T}\right)$ des dividendes futurs est alors diminuée par rapport à la valeur précédemment considérée (correspondant à $T=0$ ), soit $V\left(S, 0, Z_{0}\right)$, tandis que le niveau $Z_{T}$ de la barrière reflexive est placé plus haut que $Z_{0}$.

1) Notamment ,Dynamic decision problem in an insurance company”, ASTIN Bulletin, Mai I968, p. I I8-I3I.

2) ,The rescue of an insurance company after ruin", ASTIN Bulletin, Mai ro69, p 280-292.

3) G. Croset - Thèse: ,Quelques applications de la théorie de la décision à l'assurance dommages", Résumé à paraître dans le Bull. I.A F Juin I970. 
2.2. Il reste à considérer le second problème, c'est-à-dire, finalement, celui de l'opportunité du placement des capitaux dans une société d'assurances dommages. Cela nécessite que soit défini un critère de choix des placements.

3. Dès lors que l'on dispose dans une entreprise d'un capital $S$, il est normal de chercher à en tirer le bénéfice, ou, plus exactement, le profit actualisé maximum. C'est d'ailleurs le critère que recommande M. P. Masse en matière de choix des investissements ${ }^{1}$ ) et celui-là même qu'utilise M. Karl Borch pour définir la meilleure stratégie de l'assureur. Toutefois, le problème posé n'est pas ici exactement le même; il s'agit du choix des placements par le capitaliste. Ce dernier compare le profit avec le montant du capital misé, et, de plus, 1 tient compte du caractère certain ou aléatoire du revenu. On est ainsi conduit à envisager, avec MM. Dethoor et Grosbillot ${ }^{2}$ ), deux autres critères: celui, classique, du taux de rentabilité interne, et celui de ,l'indice de profitabilité", que l'on définira ensuite.

4. Le taux de rentabilité interne est une notion très utile, mais, en vérité, très malcommode sur le plan opérationnel.

4.1 On note tout d'abord que la décision de placer $S$ dans une compagnie d'assurance n'est économiquement sensée que si $V(S, T, Z)>S$, du moins dans l'hypothèse où le taux d'actualisation choisi correspond au taux d'intérêt normal $i$ sur le marché financier. Lorsque l'inégalité est satisfaite, le taux de rentabilité interne est supérieur ${ }^{3}$ ) au taux d'intérêt $i$. Pratiquement, il faut envisager deux éventualités.

4.1.1. Lorsque $Z>T$, l'inégalité, même considérée au sens strict, est toujours vérifiée.

En effet quelle que soit la valeur de $S>Z$, on a $V(S+d S, T, Z)$ - $V(S, T, Z)>d S$ - inégalité que l'on peut encore écrire $V(S+$ $d S, T, Z)-S-d S>V(S, T, Z)-S-$ puisque, capitaliser

1) P. Masse ,Le choix des investissements", Dunod I964.

2) Dethoor - Grosbillot ,La vie des équipements”. Dunod I967.

3) On rappelle que, par définition, le taux de rentabilité interne est le taux d'actualisation qui assure l'égalité de la valour actuelle des sommes perçues et des capitaux investis. On montre alors facilement le résultat indiqué. 
$d S$ augmente davantage $V$ que distribuer $d S$. L'inégalité devient égalité lorsque $S=Z$, on a alors $V(S+d S, T, Z)-S-d S=$ $V(S, T, Z)-S$. Ce qui signifie que, le bénéfice net de l'opération, actualisée au taux $i$, n'est pas modifié lorsqu'on ajoute $d S$. Autrement dit, le taux $i$ est le taux marginal de rentabilité de $S$ pour $S=Z$.

De l'inégalité $V(S+d S, T, Z)-S-d S>V(S, T, Z)-S$ pour $S<Z$, on déduit que le taux marginal de rentabilité de $S$ est supérieur à $i$ pour $S<Z$. Or, sans qu'on ait pu le démontrer avec précision pour $T>0$, il semble que le taux de rentabilité moyen de $S$ soit supérieur au taux marginal de $S$, donc $V(S, T, Z)>S$ pour $Z>T$.

4.1.2. Lorsque $Z<T$, il existe, en supposant une propriété de continuité, un voisinage de $Z$ où l'inégalité $V(S, T, Z)>S$ est encore vérifiée. Si $T$ est à l'intérieur de ce voisinage, les capitalistes peuvent encore accepter de laisser leurs capitaux dans l'entre prise d'assurance; dans le cas contraire, un désinvestissement est à craindre ${ }^{1}$ ).

4.1.3. Les résultats précédents montrent que l'introduction d'un capital minimum $T$ modifie fondamentalement le problème. En effet, si $T=0$ et si le chargement de sécurité inclus dans la prime commerciale est positif, alors $V(S, o, Z)$ est toujours positif, en particulier le taux de rentabilité est infinipour $S=0$; il y a donc un voisinage de zéro où $V(S, 0, Z)>S$. Par conséquent, c'est de l'existence d'un capital minimum $T$, conjuguée avec un taux de chargement insuffisant, que peut résulter une impossibilité économique d'obtenir des capitaux pour l'assurance.

4.2. Malgré l'intérêt de cette notion de taux de rentabilité interne, on doit renoncer à l'utiliser en pratique pour les raisons suivantes:

4.2.1. On peut tout d'abord remarquer que substituer au taux $i$, le taux de rentabilité interne, suppose, implicitement, que l'on puisse placer les sommes perçues sous forme de dividendes dans les mêmes conditions que le placement considéré lui-même, ce qui est loin d'être certain ${ }^{2}$ ).

1) Cette crainte n'est pas vaine, du moins en France.

2) Voir J. Urtasun "Contribution à l'étude actuarielle des opérations financières" Bull. I.A.F. no 234-235. 
4.2.2. Dans le problème considéré, se pose une question plus immédiate: doit-on prendre la valeur $Z_{T}(i)$ qui optimise $V(S, T, Z)$ avec le taux d'actualisation $i$ ou doit-on adopter la valeur $Z_{T}(x)$ qui optimise $V(S, T, Z)$ lorsqu'on substitue à $i$ le taux de rentabilité inconnu $x$ ? On sait que, dans cette dernière hypothèse, $Z$ est nécessairement inférieur à $T$ (voir 4I3), cela signifie que le capital $S$ devrait être supérieur au niveau de la barrière reflexive $Z$, ce qui n'est pas une situation normale.

Dans la première hypothèse (conserver $Z_{T}$ correspondant à $i$ ), la définition du taux de rentabilité utilisée n'est pas cohérente parcequ'elle fait toujours référence au taux $i$ du marché financier. Aucune des deux solutions proposées ne parâ̂t donc satisfaisante.

4.2.3. Le taux de rentabilité interne présente un autre inconvénient: ce n'est pas l'espérance mathématique des taux de rentabilité correspondant aux divers cheminements possibles, mais simplement le taux de rentabilité qui assure l'équivalence entre le capital investi et les profits espérés. Cela est à l'origine d'une difficulté fondamentale lorsqu'on envisage, ce qui est le cas en assurance, des revenus aléatoires.

5. Le taux de profitabilité ou taux d'enrichissement relatif est défini par le rapport $\frac{V(S, T, Z)-S}{S}$. Cette quantité paraît constituer un critère de choix susceptible d'expliquer le comportement du capitaliste.

5.1. On note tout d'abord que si le capitaliste ne se référait qu'à l'espérance mathématique du taux de profitabilité, alors il faudrait que la société soit créée avec un capital égal au minimum $T$ 1). En effet, $\frac{V(S, T, Z)}{S}$ est, comme $\frac{d V(S, T, Z)}{d S}$, une fonction décroissante de $S$. Cette dernière propriété, toujours vérifiée avec des lois de distribution du risque quelque peu vraisemblables, est intuitivement évidente: le gain $d V$ induit par une augmentation de $S$ a pour origine la réduction de la probabilité de ruine, réduction

1) Il existe une méthode directe pour établir ce résultat, voir Dethoor ,, Au delà de la programmation dynamique" Revue économique, Mai I969, pages $5 \times 5-535$. 
d'autant plus faible que, pour un même $d S$, la valeur de $S$ est plus grande.

5.2. Lorsque le revenu n'est pas certain mais aléatoire, on sait qu'il faut tenir compte aussi de la dispersion du revenu ${ }^{1}$ ). Toutefois, plutôt que d'introduire un deuxième critère, on préfère, comme $M$. Moeseke ${ }^{2}$ ), exiger que la probabilité que le taux de profitabilité soit inférieur à $\theta$ ne puisse être supérieure à $\alpha$. Autrement dit, le capitaliste souhaite obtenir un taux de profitabilité, en moyenne, le plus élevé possible, mais sans risquer trop pour cela de n'obtenir, dans certains cas, qu'un taux de profitabilité très faible. A quel niveau doit-on, dans ces conditions, fixer le capital $S$ ? Cela conduit à étudier la distribution de probabilité du taux de profitabilité.

5.2.1. On remarque tout d'abord que lorsque le capital $S$ est voisin de $T$, il y a certes une bonne probabilité que le taux de profitabilité soit élevé (comme en atteste la valeur de l'espérance mathématique de ce taux de profitabilité) mais il y a aussi une assez forte probabilité que la société doive cesser ses opérations dans un proche avenir ${ }^{3}$ ); dans cette éventualité, le taux de profitabilité est faible, voire nul.

5.2.2. Lorsque le capital $S$ est sensiblement plus grand que $T$, le taux de profitabilité est rarement très élevé (puisque le capital à rémunérer est important) mais, il y a peu de chances que la société doive cesser ses opérations (la probabilité de ruine est faible), par conséquent, le taux de profitabilité ne risque guère d'atteindre la valeur zéro.

5.2.3. A partir des remarques précédentes, on peut tracer les courbes des probabilités cumulées correspondant à la distribution du taux de profitabilité en fonction du capital $S^{4}$ ):

1) Markowitz „Portfolio selection” Journal of Finances 7, 1952, p 77-9I

2) Voir Moeseke, P V, , Stochastic linear programming" Yale economic essays 5, I965, 196-254

$\left.{ }^{3}\right)$ Il est toujours possible de renflouer la société, toutefors cela n'est qu'un palliatif, car le taux de profitabilité reste faible compte tenu de la charge du renflouement

4) Les relations de la programmation dynamique ne permettent pas d'obtenir directement ces distributions de probabilité en revanche, on peut, (n valuant chaque arc du graphe par les puissances successives du bénéfice distribué, obtenir les différents moments de ces distributions, ce qui permet 


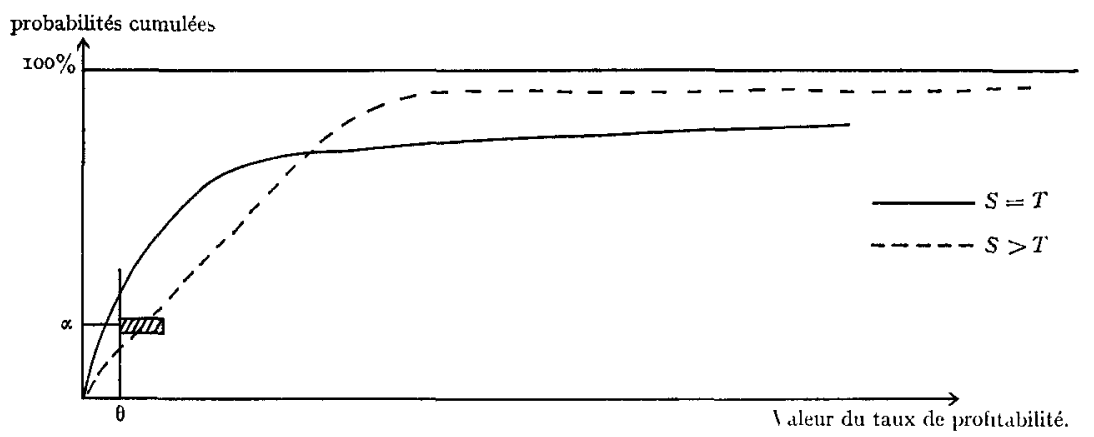

figure $I$

Les valeurs de $S$ admissibles correspondent aux courbes qui rencontrent la droite d'ordonnée $\alpha$ au delà de $\theta$. Si les exigences exprimées pour les valeurs de $\alpha$ et de $\theta$ ne sont pas excessives, on trouvera plusieurs valeurs de $S$ qui seront admissibles et on choisira, parmi celles-ci, la plus petite, elle correspond au taux de profitabilité moyen le plus élevé, tout en satisfaisant à la condition de sécurité.

6. Conclusion. Faute d'établir une distinction claire entre le capitaliste et l'assureur, on conçoit mal que certaines opérations (création de holdings notamment) aboutissent souvent à réduire le capital ou que les augmentations de capital par apport soient exceptionnelles, sauf dans le cas d'un renflouement. Certes, la distinction est rendue difficile par le fait que l'assureur-gestionnaire a généralement aussi des intérêts capitalistes dans l'entreprise, mais une partie importante des capitaux investis dans l'assurance est entre les mains de personnes qui ne sont pas les gestionnaires. Le schéma proposé, dont il faut souligner qu'il ne se réfère pas à la probabilité de ruine, semble rendre compte convenablement du comportement des capitalistes non gestionnaires, seulement préoccupés de rentabilité.

pratiquement de déterminer les distributions. Voir: M. Frechet „,Recherches théoriques modernes sur les probabilités" Traité de calcul des probabilités et de ses applications"', Tome I — Fascicule 3 (Gauthicr — Villars I950). 